\title{
Escribir para publicar: Una experiencia con grupos de escritura de investigadores universitarios
}

\author{
Writing to publish: An experience with university researcher writing groups
}

\author{
Elisabeth Rodas-Brosam $^{\text {* }^{*} \text { (D) } \text { Laura Colombo }^{2} \text { (D), María-Daniela Calle }}{ }^{1}$ (D), Guillermo Cordero ${ }^{1}$ iD \\ ${ }^{1}$ Killkana, Programa de Lectura y Escritura Académicas, Universidad de Cuenca, Cuenca, Ecuador. \\ ${ }^{2}$ CONICET, Instituto de Lingüística de la Universidad de Buenos Aires, Buenos Aires, Argentina. \\ * Autor de correspondencia: elisabeth.rodas@ucuenca.edu.ec \\ Fecha de recepción: 19 de marzo de 2021 - Fecha de aceptación: 6 de mayo de 2021
}

\begin{abstract}
RESUMEN
Las publicaciones científicas, como el medio legitimado para producir y comunicar el conocimiento, comúnmente se utilizan para evaluar la producción individual de docentes investigadores y de sus universidades. En este contexto, las prácticas de escritura relacionadas con las publicaciones científicas ameritan mayor atención para su desarrollo. Sin embargo, en Latinoamérica son escasas las iniciativas que apuntan en este sentido. Los grupos de escritura constituyen un dispositivo de desarrollo profesional-académico adecuado para enfrentar estas deficiencias. En ellos los participantes comparten borradores para que sean comentados y, mediante la colaboración y el intercambio con otros, gradualmente ajustan sus escritos a las demandas de la audiencia, un aspecto clave en la escritura académica-científica. Este estudio de caso indagó el punto de vista de los participantes de tres grupos de escritura para explorar cómo estos grupos apoyan el progreso de los escritos para su publicación. El análisis de 10 entrevistas semiestructuradas indicó que esta iniciativa beneficia a sus participantes en tres áreas principales. Primero, aprender a dar y recibir retroalimentación en un espacio seguro y de bajo riesgo ayudó a perder el miedo a esta actividad de referato inherente a escribir para publicar. Asimismo, la posibilidad de recibir comentarios de una audiencia real y variada permitió mejorar los borradores. Finalmente, las reuniones sostenidas a través del tiempo motivaron a los participantes a marcar un espacio en sus agendas y establecer un ritmo para su escritura; a su vez, hacer espacio para escribir puso de relieve la falta de asignación horaria para cumplir con esta actividad.
\end{abstract}

Palabras clave: Escribir para publicar, círculos de escritura, producción científica, investigadores, profesores.

\begin{abstract}
Scientific publications, as the means through which the academy generates and communicates knowledge, are commonly used both to evaluate the individual production of university research professors as well as that of higher-level institutions. In this context, writing-for-publication practices gain importance and deserve greater attention for their development. However, in Latin America there are few initiatives that support this situation. Writing groups constitute an adequate professional-academic development tool to face these deficiencies. Within them participants share drafts for their peers to comment on and, through collaboration and exchange with others, authors gradually adjust their writing to the demands of an audience, a key aspect in academic-scientific writing. This case study investigated participants' perspectives to explore how writing groups support the progress of writing for publication. The analysis of 10 semi-structured interviews with participants indicated that these groups benefit their participants in three main areas. First, learning how to give and receive feedback in a safe and low-risk space helped members to lose the fear connected to this activity inherent in these literacy practices. At the same time, the possibility of receiving reading impressions from a real and varied audience allowed them to improve their drafts for better comprehensibility. Finally, sustained meetings over time motivated participants to mark a space in their agendas and establish a rhythm to their writing; in turn, making room to write highlighted the lack of time faced by research professors.
\end{abstract}

Keywords: Writing to publish, writing circles, scientific production, researchers, faculty.

\section{INTRODUCTION}

Se les dice a los investigadores: escriba. Pero ¿cómo? ¿cuándo? [risas] O sea, ¿cómo es eso de "escriba"? ¿Simplemente me siento a escribir y ya? Y sabemos que obviamente esto no es así, ¿no?... El proceso de redacción no hay otra forma de conocerlo que al hacerlo $y$ 
compartiéndolo con gente que ya lo ha hecho [Miembro de un grupo de escritura].

La expectativa para los docentes universitarios en la actualidad no sólo es que ejerzan la docencia, sino que también investiguen (Lock et al., 2019; Sutherland, 2018). En su rol como investigadores, precisan realizar publicaciones que den cuenta de su actividad investigativa (Altbach, 2013; Lee, 2013; Habibie \& Hyland, 2019; McGrail et al., 2006; Nygaard, 2015), cuyo récord se ha vuelto indispensable para establecer una carrera académica, recibir promociones, obtener becas y otros privilegios (Aitchison \& Guerin, 2014).

En el caso del Ecuador, las publicaciones científicas, además de contribuir con el perfil académico de sus autores, tienen un rol central al vincular las investigaciones del sector académico con el desarrollo de la sociedad (Plan Nacional de Desarrollo, 2017-2022). El Art. 160 de la Ley Orgánica de Educación Superior (LOES) establece que es responsabilidad de las universidades la formación profesional de sus profesores e investigadores con el fin de aportar al desarrollo de la sociedad (2010). Así mismo, el Reglamento de Carrera y Escalafón del Profesor e Investigador del Sistema de Educación Superior del país, en su Capítulo II, a partir del Art. 29 señala, entre otros requerimientos, la publicación de obras de relevancia o artículos indexados como requisito para ingresar y ascender en dicho sistema (Consejo de Educación Superior, 2017).

Si bien desde la implementación de leyes relacionadas con el desarrollo de la investigación y la producción científica en el Ecuador ha habido un incremento considerable en el número de publicaciones de las universidades ecuatorianas en el período 2009-2013 (Rivera-García et al., 2017), esta contribución científica sigue siendo baja comparada con la de otros países de la región y el mundo (Morales, 2013). Casa adentro, este problema se acentúa en instituciones, como la Universidad de Cuenca, que se encuentran en un proceso de transición de universidad de docencia a universidad de docencia con investigación (Feyen et al., 2016). Adicionalmente, en la actualidad las universidades públicas enfrentan el riesgo de disminución de fondos dedicados a la investigación debido a la pandemia generada por el COVID-19 (Feyen, 2020), lo que sin duda tendrá repercusiones en el número de publicaciones científicas y la promulgación de sus resultados.

A pesar de la antedicha centralidad de las publicaciones científicas, los investigadores formados y en formación suelen encontrarse con limitadas intervenciones orientadas a la enseñanza de las prácticas letradas que rodean el escribir para publicar (Antoniou \& Moriarty, 2008; Bautista et al., 2014; Gomez-Nashiki et al., 2014). En ciertas universidades (principalmente norteamericanas y europeas) se ha apoyado a los docentes ofreciéndoles talleres, tutorías y grupos de escritura (McGrail et al., 2006). Sin embargo, en la mayoría de las instituciones continúa siendo un área olvidada (Habibie \& Hyland, 2019) ya que existe la idea equivocada que "todos los académicos saben cómo escribir para publicar y lo hacen excepcionalmente bien" (Olszewska \& Lock, 2016, p. 133).

En Latinoamérica, las acciones para el desarrollo de la escritura científica de los docentes son aún menos comunes que en el ámbito anglosajón y, si las hay, por lo general son implementadas en forma esporádica (e.g., talleres de corta duración) o en el marco de programas de posgrado (Chois-Lenis et al., 2020; Colombo, 2013; Navarro, 2017). En este contexto las escasas experiencias de docentes que se han reportado para dar apoyo a la escritura de textos para su publicación están relacionadas con grupos de escritura (e.g., Colombo \& Rodas, 2020; Márquez-Guzman \& Gómez-Zermeño, 2018; RodasBrosam \& Colombo, 2018).

Si bien los grupos de escritura se pueden implementar de diferentes formas, su labor gira alrededor de los proyectos de escritura (individuales o colectivos) de sus miembros que, por lo general, se reúnen de forma continua, durante un período de tiempo y con un objetivo común (Aitchison \& Guerin, 2014). Este objetivo puede consistir desde escribir en forma colaborativa hasta solo compartir la tarea de revisión o el espacio en el que se escribe (Haas, 2014). Estos grupos parecen constituir una buena opción para materializar el necesario acompañamiento para el aprendizaje de las prácticas del escribir para publicar (Wilson \& Cutri, 2019).

Específicamente, los grupos de escritura para docentes investigadores en formación o experimentados parecen facilitar en sus participantes el desarrollo de su identidad como escritores académicos (e.g., Bosanquet et al., 2012; Carr et al., 2020; Fajt et al., 2013; Hicks et al., 2013) ya que en estos espacios no sólo se discuten los textos sino también cómo participar mediante los escritos en las discusiones del campo. Otro aspecto positivo es la posibilidad de tener un espacio seguro donde ejercer el referato y ganar experiencia en cuanto a esta práctica letrada relacionada con el escribir para publicar (e.g., Bosanquet et al., 2012; Fallon \& Whitney, 2016; Galligan et al., 2003). Al mismo tiempo, pertenecer a un grupo de escritura proporciona un espacio de interacción social con otros que también están experimentando situaciones similares con respecto a su desarrollo escritural y las demandas asociadas con su rol como investigadores (e.g., Allen, 2019; Cassese \& Holman, 2018; Grant, 2006; Lock et al., 2019; Plummer et al., 2019). Por otro lado, un objetivo común y las interacciones que se propician dentro de estos grupos desarrollan un compromiso y un sentido de mutualidad entre sus miembros, donde se busca el beneficio de todos en este espacio (e.g., Carr et al., 2020; Hicks et al., 2013) Finalmente, aquellos grupos de escritura con miembros provenientes de diferentes disciplinas proporcionan una variedad de miradas y comentarios sobre los textos que pueden, en última instancia, contribuir a hacer el escrito más accesible para un público fuera del área disciplinar del autor (e.g., Colombo \& Rodas, 2020; Dwyer et al., 2012; De Feo et al., 2016; Plummer et al., 2019).

Teniendo en cuenta el rol central de las publicaciones en la sociedad a nivel mundial y la escasez de iniciativas orientadas a acompañar a los docentes universitarios de forma sostenida con la intención de mejorar sus prácticas de escritura, el presente trabajo presenta algunos resultados de un proyecto de investigación que analiza el funcionamiento de grupos de escritura de investigadores en la Universidad de Cuenca. En esta ocasión, específicamente, presentamos los hallazgos relacionados con las percepciones de sus miembros con respecto a la utilidad de estos grupos para el desarrollo profesionalacadémico y el mejoramiento de la producción científica de los profesores y/o investigadores. 


\subsection{Los grupos de escritura: implementación y participantes}

En el 2017, a través de Killkana: Programa de Lectura y Escritura Académicas de la Universidad de Cuenca, se comenzó a coordinar grupos de escritura. El objetivo principal fue dar apoyo a los docentes investigadores (en formación o experimentados) y a asistentes de investigación en cuanto a la escritura y publicación de artículos científicos. Cada grupo estuvo conformado por tres o cuatro miembros. Las reuniones presenciales se sostenían en las oficinas del Programa para crear un ambiente donde los participantes pudieran desconectarse de alguna manera de sus otras actividades y evitar posibles interrupciones (Murray, 2013). En este contexto, la primera autora actuó como coordinadora y observadora participante, asistiendo a todas las reuniones de los distintos grupos, para lo cual contó con horas asignadas en su distributivo ${ }^{1}$. Su rol consistió en acordar con los participantes la agenda para sostener las reuniones presenciales, determinar los textos a comentar antes y durante cada reunión, modelar las prácticas de retroalimentación por medio de sus propios comentarios y proporcionar apoyo en la parte de la escritura académica y el inglés como lengua extranjera, en caso de ser necesario.

A pesar de que la mayoría de los participantes formaba parte de un equipo de investigación, su grado de experiencia con la escritura académica era variado y su participación en el grupo de escritura, sin estar considerado dentro de su carga horaria como docentes investigadores, se realizaba de forma voluntaria y fuera del horario laboral. Al momento del estudio, los tres grupos de escritura analizados venían funcionando por más de dos semestres. Las reuniones se realizaban cada siete o quince días y duraban entre 30 y 60 minutos. La mayoría de los participantes provenían de diferentes disciplinas de las ciencias duras.

\section{METODOLOGÍA}

Este estudio exploratorio cualitativo (Maxwell, 2013) analiza las percepciones de los miembros de tres grupos de escritura de docentes investigadores y ayudantes de investigación implementados en la Universidad de Cuenca, a partir de 10 entrevistas semiestructuradas.

Para las entrevistas semiestructuradas se utilizó un muestreo intencional (Creswell, 2007) al considerar que todos los miembros de los grupos de escritura (12 en total) estaban en la misma posición de proporcionar información relevante y perspectivas variadas sobre el objetivo del estudio. De los doce miembros, diez aceptaron participar de la entrevista (83.3\%). Para esto, recibieron información del estudio y firmaron un consentimiento informado. Con el objetivo de mantener la imparcialidad en la recolección de datos, las entrevistas fueron conducidas por dos miembros del equipo de investigación desconectados y desconocidos por los entrevistados. La entrevista estuvo compuesta de 14 preguntas que buscaban indagar sobre las percepciones de los participantes acerca de su

\footnotetext{
${ }^{1}$ La coordinadora contó con asignación horaria desde el semestre marzo-agosto 2017 hasta el semestre marzo-agosto 2019. A partir del semestre septiembre 2019-febrero 2020 a la fecha de publicación de este artículo, la actividad de coordinación se ha
}

participación en los grupos de escritura; estas duraron entre 25 a 40 minutos. Para mantener el anonimato y confidencialidad de los datos se han asignado seudónimos a los participantes.

Para llevar a cabo el análisis interactivo de datos (Maxwell, 2013), primero se realizaron lecturas recurrentes de las transcripciones de las entrevistas. Luego, se identificaron las unidades de información que fueron abordadas por medio de procedimientos analíticos tales como "marcar lo que es de interés en el texto" (Seidman, 2006, p. 117), esto relacionado con la percepción de los miembros sobre su participación en los grupos de escritura. Una vez marcados los segmentos de interés, las unidades fueron agrupadas en categorías descriptivas que se ajustaron y redefinieron continuamente para dar cuenta de nuevas unidades (Creswell, 2007). A su vez, se llevó a cabo la categorización y la contextualización de los datos (Maxwell \& Miller, 2008), combinando procesos deductivos e inductivos. Las unidades, categorías e interpretaciones fueron discutidas por los miembros del grupo de investigación a fin de establecer confiabilidad (Denzin \& Lincoln, 2005). Los temas que emergieron se utilizan para reportar los resultados.

\section{RESULTADOS Y DISCUSIÓN}

El análisis de las entrevistas semiestructuradas con el fin de indagar la percepción de los miembros sobre su participación en los grupos de escritura indicó acuerdo de la mayoría de los entrevistados en cuanto a tres temas principales. En primer lugar, los grupos de escritura propician un espacio de bajo riesgo y seguro en dónde se aprenden, mediante su ejercicio, las prácticas de revisión entre pares, inherentes al proceso de referato de las revistas científicas. Además, ofrecen a los autores la posibilidad de recibir impresiones lectoras y retroalimentación por parte de una audiencia real, amplia y variada. En tercer lugar, organizar las fechas de las reuniones ayudó a los participantes a establecer un ritmo de trabajo e incluir dentro de sus agendas la actividad escritural. Sin embargo, esto también puso de relieve una de las mayores dificultades que los docentes investigadores enfrentan: encontrar un tiempo y espacio para escribir, revisar y enviar a publicar.

\subsection{El proceso de dar y recibir comentarios: perder el miedo}

Escribir para publicar en revistas implica, obligadamente, que el texto sea revisado por pares antes de hacerse público. Para muchos, esta experiencia puede ser desmoralizadora e intimidante si es que no se ha experimentado anteriormente. Los grupos de escritura, según nuestros participantes, proporcionan una oportunidad para ensayar de manera segura esta práctica letrada: el referato. Es al dar y recibir comentarios en forma asidua que los participantes aprenden a recibirlos

realizado de forma voluntaria al no contar con horas asignadas dentro de su distributivo para esta actividad. 
sin temor y a brindarlos con respeto y consideración. A continuación, detallamos lo que los integrantes de estos grupos resaltaron sobre esta tarea.

\section{Tomar el rol de autores: aprender a recibir comentarios}

Aprender a recibir comentarios, en cierta forma, implica perder el miedo a la crítica y poder aprovecharla para mejorar el escrito. Este aprendizaje está directamente ligado con la forma de trabajo que se lleva dentro de los grupos de escritura y que fue establecida mediante las reglas de interacción acordadas al comienzo de su funcionamiento. En estos grupos el proceso de dar y recibir comentarios involucra dos etapas. En la primera, uno de los participantes comparte su texto dos a tres días antes de la reunión presencial subiéndolo a una carpeta de Google Drive creada por la coordinadora. Allí, también previo a la reunión, los demás miembros brindan retroalimentación utilizando la función "insertar comentario". El objetivo de esta primera interacción asincrónica es que los revisores tengan tiempo para leer el texto con detenimiento y ofrezcan sus impresiones lectoras, brinden críticas y alternativas de solución a fin de que el autor mejore su borrador. Estos comentarios, lejos de ser imposiciones, consisten en sugerencias para el autor. El segundo momento del proceso de revisión se da en las reuniones cara a cara, donde los integrantes revisan, explican, expanden y discuten los textos y la retroalimentación ofrecida por los pares.

Por tanto, las reuniones de los grupos de escritura analizados consisten, principalmente, en intercambios basados en los textos en proceso y los comentarios realizados por quienes lo leyeron. Para Tatiana, quien tenía cierta experiencia en la escritura de artículos científicos por medio de su maestría y el equipo de investigación del que era parte, los comentarios tanto orales como escritos que se reciben en los grupos de escritura circulan en un ambiente donde está bien equivocarse ya que es parte del proceso de construcción del texto. Según ella, en las reuniones se daba la siguiente dinámica:

$$
\begin{aligned}
& \text { Es una conversación ligera en el sentido que } \\
& \text { uno no se siente juzgado, ni nada, sino } \\
& \text { realmente yo en mi grupo sentí que eran aportes } \\
& \text { y una crítica constructiva. Entonces, como que } \\
& \text { corre el riesgo además de equivocarse todo lo } \\
& \text { que pueda al principio, porque luego se va } \\
& \text { corrigiendo y tomando en cuenta y leyendo lo } \\
& \text { que sugieren los compañeros y lo que se habla } \\
& \text { en el grupo. }
\end{aligned}
$$

Para muchos investigadores, tanto novatos como expertos, presentar textos en proceso conlleva un gran esfuerzo ya que mostrar algo que uno considera como no terminado suele implicar cierto grado de incomodidad. Esta situación está ligada al falso ideal del escritor solitario que produce sus textos sin ningún problema o ayuda ya que posee un don innato (Gere, 1987). Este falso ideal hace que nos sintamos "malos" escritores al compararnos con una imagen poco realista del proceso de escritura que lo muestra como algo lineal, simple y sin pormenores. Por el contrario, el proceso de escritura es intrincado y laborioso hasta para los escritores más experimentados (e.g., Rijlaarsdam \& van den Berg, 2006). Pero como rara vez podemos ser testigos de ello (Castelló, 2007), muchas veces pensamos que el problema es nuestro.
Los grupos de escritura precisamente abren un espacio donde combatir este falso ideal de que la escritura es un don poseído por pocos, ya que permiten presenciar y compartir los avatares del proceso de escritura con otros. En esta línea es que, según declararon la mayoría de los participantes, los grupos de escritura ayudan a perder el miedo a exponerse. Isabel, quien antes de ser parte de los grupos principalmente había recibido comentarios de su director de tesis o su director de proyecto de investigación, comentó que el escritor que presenta un borrador en un grupo de escritura tiene que quitar de su mente que los lectores "le van a acabar [le van a criticar todo] y que van a pensar que el texto que uno tiene tal vez esté mal escrito". De hecho, según Alicia, quien se describió a sí misma como una escritora novata quien comenzaba este proceso de aprendizaje de escribir para publicar, los grupos de escritura ayudan a dar el primer paso para perder el miedo a compartir un texto: simplemente hacerlo. En este sentido indicó:

Quiera o no, usted ahí [en el grupo de escritura] da su primer paso, pierde ese miedo o ese temor a que cómo voy a escribir, qué dirán de mi artículo: es muy básico, es muy simple no estará para una revista. Entonces, yo creo que ahí no es que se aprende de la noche a la mañana. $O$ sea, yo ya estoy en el grupo un año y medio creo y todavía tengo mis falencias, y sigo teniendo mis falencias... pero yo les he dicho eso [a las personas que recomienda que participen en los grupos], o sea que vayan, que pierdan ese miedo porque ahí es donde vamos a poder aprender a escribir, porque las cosas se aprenden haciendo.

Al perder el miedo de presentar sus textos en construcción, los autores comienzan a aprovechar el potencial de los comentarios de sus compañeros para mejorar sus textos. Es así como, para Clara, el grupo de escritura era el lugar ideal para poner a prueba su texto y mejorarlo hasta que lo consideraba listo para que otros lectores, fuera de su círculo seguro, lo revisaran y evaluaran. En sus palabras:

Lo que he hecho es tener los comentarios de todos los compañeros del grupo de escritura, para posteriormente ahí sí enviar el avance de mi texto a, por ejemplo, en este caso, a mi directora. Ella tiene muchísima experiencia [en el área disciplinar]. Entonces yo realmente me caigo y me levanto en el grupo de escritura para luego de ahí aventurarme a mandar el artículo. Entonces sí, más bien es como un borrador que se envía [a los miembros del grupo] para que sea de fácil comprensión y luego yo envío a la persona que tiene más experiencia todavía, y es enfocada en el tema... Mis borradores están aquí en el grupo de escritura. Una vez que esté bien redactado, bien comprensible [para los compañeros del grupo de escritura] se envía [a otros].

Estos dos aspectos de aprender a recibir comentarios (i.e., perder el miedo a exponerse como autor al compartir borradores y aprovechar los comentarios para mejorar el texto) fueron considerados una gran ventaja por varios de los participantes. En efecto, los grupos de escritura permiten a los integrantes ensayar usos específicos del lenguaje junto con otros y así, gradualmente, aprender cómo dirigirse a una audiencia en forma efectiva. A la vez, todo esto se da en lo que al cabo de un breve tiempo se 
comienza a percibir como un espacio seguro, donde las críticas son respetuosas y constructivas. Con relación a esto, Lili marcó que los grupos de escritura precisamente estaban para acompañar ese proceso de composición que no sucede de un día a la noche. Según ella, un consejo que le daría a alguien que fuera a participar en un grupo de escritura por primera vez sería el siguiente:

No te preocupes de que no sea perfecto, [que] no parezca versión final. Escribe primero, escribe lo que salga y vamos construyendo [en el grupo de escritura] desde ahí... a no tomarse las críticas personales sino más bien como que es para una mejor construcción del trabajo.

La frase "vamos construyendo" denota el acompañamiento en la escritura y el colaborar con otros a fin de mejorar los borradores que se trabajan en los grupos. Esta característica es de especial importancia ya que permite a los participantes desarrollar confianza en sí mismos como escritores, especialmente aquellos que son más novatos en el mundo de las letras académicas.

\section{Tomar el rol de revisores: aprender a dar comentarios escritos $y$ orales}

En los grupos de escritura además de aprender a recibir retroalimentación para mejorar los escritos, los participantes parecen comenzar a perder el miedo no sólo a la crítica, sino a dar comentarios. Esto se da gracias al hecho de que, en los grupos, efectivamente, se ejerce el rol de revisor de textos, un rol crucial en el proceso de publicación de artículos científicos. Esto permite que los integrantes aprendan de manera situada los usos de las prácticas letradas (Colombo, 2017). Sin embargo, a diferencia de las evaluaciones por pares que se dan comúnmente en el circuito de publicación, en los grupos de escritura que analizamos, como mencionamos anteriormente, la retroalimentación se brinda en dos tiempos. En un primer momento se intercambian comentarios escritos antes de las reuniones presenciales para luego, durante las reuniones, sostener intercambios orales en los cuales se aclaran, expanden o discuten tanto los textos como los comentarios escritos.

Esta segunda instancia en la que se discuten los comentarios permite a los autores entablar diálogos con los demás integrantes (sus lectores-prueba) e indagar un poco más sobre el feedback recibido. Por ejemplo, Lara, quien ingresó al grupo de escritura por recomendación de su directora del grupo de investigación, recalcó este aspecto de los grupos en la entrevista: "En mi caso, me comentaban algo [por escrito] y no entendía por qué ponían eso. Cuando estábamos en la reunión, allí me explicaban." Para todos los entrevistados, los dos momentos de retroalimentación eran necesarios y encontraban especialmente enriquecedor poder dialogar sobre los comentarios escritos. Mateo, con varios años de experiencia en escribir para publicar, por ejemplo, declaró lo siguiente en cuanto a esta forma de organizar la retroalimentación:

Definitivamente [las reuniones presenciales] son claves. Uno siempre cuando lee comentarios que han hecho a tu trabajo tiene la tendencia de leerles con el peor tono mental posible, ¿no? Te imaginas la voz de la otra persona diciendo: jesto está mal escrito!, jeste verbo no va aquí! [se ríe] Cuando tienes la interacción humana, te das cuenta de que precisamente esos comentarios están ahí para ayudar y la presencia y el tono de voz y la cercanía humana ayudan muchísimo, por un lado, a manejar esos comentarios y segundo, para entender con mayor claridad de qué se trata el comentario o el feedback.

Como muestra el anterior fragmento, los encuentros cara a cara ayudan a los participantes a construir comunidad y a humanizar los comentarios. Esto, a la vez, contribuye a que los revisores observen cómo su retroalimentación es recibida, algo que rara vez sucede en el mundo académico, donde las devoluciones son por escrito por parte de los evaluadores de artículos enviados a revistas científicas. Además, la oportunidad de intercambios orales con quienes fungieron como lectores prueba del texto enviado al grupo de escritura abre un espacio para que los revisores comuniquen en forma más clara y completa su experiencia de lectura, tal como afirmó Leo:

Sí, las reuniones son totalmente importantes porque en el texto, o sea, es como cuando escribimos en un correo o cuando escribimos a través de una red social. No está totalmente claro. Quizá está el mensaje como tal pero no está la forma en que le decimos [el tono de voz]. Entonces, la retroalimentación in situ es totalmente importante. $O$ sea, hubo este comentario que no me quedó claro, entonces, ¿me puede explicar? Una sola revisión se vuelve totalmente impersonal y estamos trabajando con personas. Somos humanos y necesitamos, por ejemplo, relacionarnos en ese aspecto.

Desde la perspectiva de los entrevistados, entonces, uno de los primeros consejos para nuevos participantes sería tener en cuenta cómo se dan los comentarios, justamente en referencia al tono que se emplea al revisar los textos en las reuniones presenciales. En relación con esto, varios de los miembros indicaron que al dar retroalimentación es necesario pensar en el otro, evitar lo que Isabel denominó "los hachazos," es decir, dar críticas poco constructivas o de mala manera, especialmente cuando se tiene en cuenta que éstas pueden herir susceptibilidades e ir en detrimento del objetivo principal de la revisión entre pares: ofrecer material para revisar y mejorar el texto. Así lo confirmó Alicia al mencionar aquello que le diría a alguien que va a comentar un texto en el marco de un grupo de escritura por primera vez:

Primero, sea prudente, no vaya a herir susceptibilidades. Que siempre diga las cosas [los comentarios] con el respeto que se merecen [los compañeros]. Trate de manejar mucho la modalidad de la voz. O sea, trate de llegar a la otra persona, ¿sí?

En esta misma línea, para otro participante, los comentarios que se dan a los textos bajo revisión deben ser siempre con el objetivo de construir. Por tanto, se espera que los revisores no solo señalen los problemas del escrito, sino también mencionen aquello que creen está bien. Así, existe la motivación de dar retroalimentación positiva y ofrecer, si es posible, soluciones (Johnson et al., 2017). Al respecto, Mateo mencionó lo siguiente:

Los comentarios definitivamente deben ir en un sentido muy constructivo, centrándose en proveer cosas que realmente sean de ayuda... Tratar de dar feedback positivo en el sentido de 
resaltar también las cosas que están bien y que nos han gustado y no solamente corregir lo que creemos que no está bien. Porque eso también ayuda mucho. Es decir, esa parte les gustó a todos, entonces no la voy a tocar.

\section{El compromiso mutuo como un regalo}

Dar comentarios conlleva un compromiso que se adquiere al momento que cada integrante decide ser parte de un grupo de escritura ya que los autores tienen la expectativa que el aporte de sus lectores ayude a mejorar su borrador. Esto implica que es necesario que los participantes inviertan suficiente tiempo para leer con detenimiento y comentar los textos y hasta, en ocasiones, involucrarse de forma periférica con el tema, tal como lo indicó Tatiana: "si no entendíamos algo del artículo que el compañero o la compañera nos estaba compartiendo, sí nos llevaba, nos incentivaba a buscar tal vez en Internet para ver de qué estaba hablando" para poder dar mejores comentarios. Este compromiso con el compañero también lo mencionó Andrea:

Creo que cuando ingresa [un nuevo miembro] como integrante del grupo de escritura debe tener un compromiso bastante grande. Hay que dedicar el tiempo suficiente para leer con mucha minucia los artículos... Le diría a esa persona que realice los comentarios como si fuera de una revista para que sea el aporte. De eso se trata. Si uno entra en estos grupos, uno quisiera que todas las personas que leen nos den la mayor cantidad de comentarios en vista de mejora. Entonces, si es que uno tiene el mismo compromiso, va a ser igualmente retribuido por las otras personas cuando las fechas sean para nosotros.

Ahora bien, esto de ser "igualmente retribuido" se debe a que, en los grupos de escritura analizados, los roles de autores y revisores son rotativos: quien ofrece retroalimentación sabe que a futuro también recibirá retroalimentación de sus pares. En palabras de Lili: "como que todos estamos en lo mismo. Entonces, si bien estoy dando una asesoría gratis, también estoy recibiendo un montón de asesoría gratis y digamos, en ese sentido, estamos todos contribuyendo y siendo retribuidos." Así, se establece una suerte de "intercambio recíproco de regalos" (reciprocal gift giving; Guerin, 2014), basado en relaciones de confianza y obligación mutuas que se complementan con el hecho de que los integrantes comparten expectativas y presiones comunes en cuanto a tener que escribir para publicar. Al respecto, Leo dio voz a los acuerdos mutuos al interior de su grupo: "Yo creo que era un tema de responsabilidad y de profesionalismo, en cierta forma, porque decimos: vamos a acordar algo [i.e., escribir y dar feedback], tenemos que hacerlo, por algo estamos en el grupo".

En definitiva, en los grupos de escritura se aprende tanto a dar como a recibir comentarios. Por un lado, aprender a recibir comentarios y utilizarlos para mejorar sus borradores permite a los participantes ir desarrollando gradualmente la confianza en sí mismos como autores. Por el otro, aprender a dar comentarios resulta algo valioso en el mundo académico donde rara vez se enseña a ejercer la función de referato en forma respetuosa y teniendo especial cuidado con el otro. Finalmente, involucrarse en la tarea de dar y recibir comentarios va forjando al interior del grupo una cultura de "intercambios recíprocos de regalos" donde el compromiso mutuo permite el crecimiento de todos y cada uno de los miembros.

\subsection{Contar con una audiencia académica real y más amplia}

A pesar de que los participantes de los grupos analizados llevan a cabo la labor de escritura entre reuniones y en solitario, luego cuentan con una audiencia real que les permite lograr una mejor construcción y calidad del texto al ayudarlos a anticipar posibles preguntas y reacciones ante el escrito. Así, la interacción con otros en tiempo real sobre el desarrollo de un borrador permite que el autor determine dónde el texto no está claro y requiere más detalles para una mejor comprensión de acuerdo con los comentarios escritos proporcionados con antelación. En este sentido, los grupos de escritura, conforme a la percepción de sus miembros, facilitan impresiones lectoras de una audiencia académica concreta, con la que se puede dialogar y acceder a perspectivas variadas.

En primer lugar, todos los miembros de los grupos de escritura mencionaron que uno de los objetivos principales de su participación era verificar que sus textos fueran comprensibles para quienes no eran expertos en la temática. Así, como lo indica Andrea, una investigadora con más de cinco años de experiencia en la escritura de artículos científicos, un aspecto positivo del grupo era que en este espacio "se podía tener una perspectiva de saber si es que nosotros estábamos escribiendo con claridad la información que tratamos de ofrecer a la ciencia. Eso era muy bueno cuando estuvimos en las reuniones presenciales, tener ese tipo de retroalimentación".

A su vez, si bien los miembros de los grupos de escritura no necesariamente eran expertos en las mismas temáticas, sí compartían el hecho de todos estar llevando a cabo algún tipo de investigación y estar todos embarcados en la producción de un artículo científico. Por tanto, los comentarios que podían aportar iban más allá de las impresiones lectoras que hubiera proporcionado un lector sin formación en investigación. En esta línea, Isabel coincidía con otros participantes en que estos tipos de intercambios obligaban a los autores a ser más reflexivos y a considerar sus textos desde otras perspectivas para hacerlos más comprensibles. En sus propias palabras:

Usted recibe comentarios de varias personas que incluso no están en su misma rama. Entonces al mostrar su texto o lo que usted tiene, ellos pueden dar su comentario o sus consejos para poder hacer que esa redacción sea entendible para todo público. O sea, no solo para el público al que usted está mostrando [el lector del área disciplinar] sino que se entienda para otras personas ya que, si usted escribe, usted piensa que es entendible para todos. Entonces es mejor recibir consejos de partes externas.

Asimismo, Tatiana también declaró considerar importante tener una audiencia real para que su texto sea más claro e indicó que los comentarios recibidos en el grupo de escritura le permitían trabajar no solo la compresibilidad sino también la estructura textual de su artículo:

Otra cosa que nos ayudaba [los comentarios dentro del grupo] es a estructurar bien el artículo, como identificar fácilmente que otra persona que lee el artículo no entiende 
realmente donde está, por ejemplo, la introducción o cuál es el objetivo. Porque a veces tendemos a enredarnos [los autores], ¿no?

El segundo aspecto ligado a recibir comentarios también mayoritariamente mencionado por los participantes fue el acceso a perspectivas diferentes posibilitado por la heterogeneidad en cuanto a la formación disciplinar de los miembros. Aunque la mayoría de ellos provenían de las ciencias duras, la diferencia entre los integrantes era suficiente como para aportar una visión por fuera de sus grupos de investigación. Así, Leo consideró que tener las lecturas de otros fuera de su disciplina le ayudó a salir de los conceptos preestablecidos y rara vez cuestionados al interior de su equipo de investigación:

Más bien el tema de compartir con otras personas es justamente para salirnos de ese cajón en el cual nos encontramos y pues abrirnos, ¿no? Exponer [el texto] a otro tipo de personas y decir, bueno, aquí resaltan estas falencias, porque de vez en cuando nos encontramos en el mismo grupo [de investigación], inclusive, tan encajonados que no salimos de allí y pensamos que todo está bien.

Para Leo, entonces, las lecturas y comentarios de sus compañeros del grupo de escritura ayudaron a ver su texto desde una perspectiva nueva. Esto va en línea con trabajos anteriores que han resaltado lo ventajoso de implementar grupos de escritura interdisciplinarios (e.g., Bosanquet $e t$ al., 2012; Colombo \& Rodas, 2020; De Feo et al., 2016; Galligan, et al., 2003; Smith et al., 2013). En efecto, para la mayoría de los entrevistados, poder trabajar con gente proveniente de otra disciplina fue considerado uno de los beneficios principales de los grupos. Tal como lo sostiene Lara en el siguiente fragmento:

$$
\begin{aligned}
& \text { Era que podíamos tener diferentes puntos de } \\
& \text { vista porque era como grupos } \\
& \text { multidisciplinarios. Entonces nos podíamos } \\
& \text { ayudar un poco de acuerdo a diferentes } \\
& \text { perspectivas. Creo que eso era lo mejor de } \\
& \text { compartir ese tiempo. }
\end{aligned}
$$

En definitiva, según los participantes, contar con una audiencia académica real, amplia y variada les resultó enriquecedor a pesar de que sus pares no conocieran en profundidad el contenido disciplinar de los extos que estaban bajo revisión.

\subsection{El tiempo: un factor fundamental}

Sin importar si son escritores experimentados o novatos, la mayoría de los académicos enfrentan tensiones relacionadas con las demandas que acarrean sus diferentes roles en la universidad, entre ellos la docencia y la investigación (Stensaker, 2017). Muchos se encuentran involucrados en proyectos de investigación para los cuales no tienen horas laborales asignadas (Grant \& Knowles, 2000; Sheridan et al., 2019). Por lo tanto, el tiempo que dedican a la escritura de artículos para publicación suele quedar relegado ante las demandas de las demás actividades. De esta manera, la escritura académica se convierte en algo que se realiza cuando se tiene "tiempo extra" y, así, va quedando gradualmente por fuera de las labores del día a día. Por esta razón, los participantes expresaron que involucrarse en este tipo de iniciativa les permitía hacer lugar a la escritura, asignando tiempo para esta actividad en sus agendas de trabajo, lo cual permite no sólo realzar su importancia sino también combatir su invisibilidad.

En primer lugar, definir quién presentará qué en las subsiguientes reuniones ayuda a cada uno de los participantes a establecer con antelación una agenda de escritura personal que respete las fechas límites para enviar el borrador a ser comentado por sus compañeros. Estos acuerdos que se dan al interior de cada grupo en cuanto a fechas y textos a ser comentados se realizan con la ayuda de la coordinadora. Para Isabel, por ejemplo, las reuniones pactadas al interior de su grupo constituían el incentivo para continuar con su proyecto de escritura, aunque fuera difícil encontrar el tiempo para hacerlo: "Sí, me gusta estar allí [en su grupo de escritura] aunque es duro porque hay que presentar el artículo en la fecha que es, pero te da la motivación para vos poderte sentar y tener a tiempo tu artículo". De igual manera, Clara explicó que para ella las fechas acordadas eran uno de los aspectos más importantes del grupo de escritura ya que le permitía establecer objetivos que la ayudaran a avanzar con su propio texto: "me llevó un poco a trabajar bajo presión. Entonces el hecho de establecer una fecha límite de presentación o el avance específico de una sección del artículo... a uno le obliga justamente a cumplir objetivos".

La organización de las reuniones, entonces, parece ayudar a que cada uno de los miembros establezcan objetivos y fechas límites, encontrando, así, un ritmo de trabajo que permite avanzar con la redacción y revisión de los textos gracias al compromiso con los demás (Hicks et al., 2013; Plummer et al., 2019). Este aspecto valorado por los participantes de los grupos de escritura también resalta lo desafiante de poder desconectarse de sus otras responsabilidades (Murray, 2013) a fin de encontrar tiempo para escribir, actividad que para muchos docentes e investigadores universitarios sigue estando en la periferia de sus labores académicas.

En efecto, todos los entrevistados mencionaron a la falta de tiempo como uno de los aspectos desafiantes en cuanto a participar en los grupos: precisamente, les costaba encontrar tiempo para avanzar con sus textos, leer y comentar los borradores de sus compañeros. Así, Mateo, quien consideraba que el grupo le daba un cierto orden y ritmo a su proceso de escritura, también percibía lo siguiente:

\section{Lo más desafiante ha sido precisamente cumplir con los tiempos, ¿no? Aunque obviamente como es un grupo muy flexible, siempre nos damos también flexibilidad. Pero tratar de cumplir con los acuerdos tanto para presentar los manuscritos propios como para revisar los de los otros siempre exige un poquito más de fuerza, de compromiso.}

Esta relación ambivalente con el tiempo también fue mencionada por otros miembros. Alicia, quien dividía su tiempo entre actividades administrativas, docentes y de investigación, resaltó que "algo muy desafiante ha sido sacar el tiempo para dedicarse realmente a escribir este artículo, ¿no?... O sea, se necesita dedicación, esfuerzo y tiempo". Para ella, esto significaba no solamente tener suficiente tiempo para escribir el texto sino también para seleccionar la revista dónde publicarlo, revisar la bibliografía, reflexionar e incorporar los comentarios de sus compañeros del grupo de escritura, entre otras acciones entramadas en el avance de su borrador. Sin 
embargo, este texto, al final de su primer año de participación en el grupo de escritura, fue enviado a la revista seleccionada y actualmente se encuentra publicado.

Como otros estudios han resaltado (e.g., Bosanquet et al., 2012; Grant, 2006), la carencia de tiempo es una de las principales razones por las que las personas abandonan los grupos de escritura. Aquellos participantes que al momento de la entrevista ya no asistían indicaron que la razón de su retiro había sido precisamente el no poder cumplir con todos los compromisos de su vida académica y personal. En este sentido, Tatiana, quien enfrentaba la demanda de su tiempo para varias actividades fuera y dentro de la universidad, remarcó lo siguiente:

Eso sí afecta [el tiempo disponible], que es muy diferente a una persona que está todo el tiempo en lo académico y no le roba tanto tiempo otra actividad para concentrarse y dedicarse muchas horas para escribir. Porque se necesita un tiempo de concentración. Entonces, empecé a fallar, justamente a llegar a los círculos de escritura y no cumplir con los plazos que eran necesarios para que el grupo pueda avanzar. Entonces preferí retirarme.

Por otro lado, para Andrea el factor del tiempo también jugó un papel clave en su participación en el grupo de escritura:

El desafio siempre fue, en general, encontrar el tiempo para cumplir con las actividades que uno se propone adicionalmente a los tiempos de trabajo que uno maneja. Y finalmente esto repercutió en el hecho de que yo no pude continuar. Son horas de trabajo que uno pone en la escritura científica que no están dentro de la carga horaria que uno tiene que cumplir.

Tal como ilustran los comentarios anteriores, los participantes enfrentan variadas demandas que compiten por el escaso tiempo disponible en sus agendas. Tan sólo en el plano laboral, muchos profesores deben dedicar tiempo a tareas de docencia y administración que restan tiempo a las actividades investigativas, inherentemente relacionadas con escribir para publicar. Para muchos, desconectarse de estas actividades que están visiblemente reconocidas en sus horarios de trabajo para conectarse con aquellas que, lamentablemente, rara vez cuentan con horas asignadas y encontrarse con otros que también luchan por encontrar un espacio para escribir para publicar (Murray, 2013) no es posible si es que esta actividad no se vuelve visible además de necesaria. Los grupos de escritura, precisamente, inauguran espacios donde esta actividad no sólo cuenta con un tiempo sino también un espacio protegido donde comenzar a ejercer, junto con otros, las prácticas letradas relacionadas con la escritura científica.

\section{CONCLUSIÓN}

Este artículo presenta parte de los resultados correspondientes a un estudio sobre el funcionamiento de los grupos de escritura para docentes investigadores y asistentes de investigación implementados en la Universidad de Cuenca. Específicamente, se determinaron las percepciones de los miembros con respecto a la utilidad de esta iniciativa para su propio desarrollo académico-profesional, concretamente en lo relacionado con la producción y publicación de escritos académicocientíficos. Los resultados obtenidos apuntan a una perspectiva positiva sobre el rol que desempeñan estos grupos en tres aspectos principales.

En primer lugar, según los miembros, ser parte de los grupos de escritura les permitió experimentar de forma real y situada el dar y recibir comentarios de pares académicos y perder el miedo a esta actividad que está directamente relacionada con el quehacer científico (e.g., Fallon \& Whitney, 2016; Galligan et al., 2003). Así, retroalimentar y recibir retroalimentación sobre borradores a fin de mejorarlos fue posible gracias al compromiso mutuo asumido al interior de los grupos. Esto dio lugar a un "intercambio recíproco de regalos" (Guerin, 2014) en un marco de confianza y compromiso entre los miembros, quienes tenían un objetivo común: avanzar con la escritura de sus textos para publicarlos en revistas científicas. Por otro lado, tener una audiencia real y variada (en cuanto a disciplinas de origen y experiencia en escribir para publicar) proporcionó a los participantes la posibilidad de mejorar no sólo la estructura de sus textos sino también considerar la perspectiva de lectores por fuera de su área disciplinar. Esto, según los entrevistados, ayudó a que los autores ajustaran sus textos para hacerlos comprensibles y fáciles de entender para un lector menos especializado y que no conociera a profundidad el tema tratado. Finalmente, el ritmo de trabajo -establecido en forma democrática y de común acuerdo con la ayuda de la coordinadora- permitió a los participantes hacer lugar en sus agendas y otorgarle mayor importancia y visibilidad al proceso de escritura. Asimismo, permitió que los participantes se desconecten de otras actividades relacionadas con la labor universitaria para abrir un tiempo y espacio específico a las tareas de redactar y revisar textos, lo que se tornó más fácil al contar con un lugar determinado y al haber asumido un compromiso con otras personas (Murray, 2013).

La implementación, seguimiento y evaluación de esta iniciativa se dio a través del Programa de Lectura y Escritura Académicas de la Universidad. En efecto, los grupos de escritura contaron con un espacio para reunirse, el cual gradualmente fue construyéndose como un espacio seguro (e.g., Carr et al., 2020; Fajt et al., 2013) donde trabajar sus textos en forma colaborativa. Así mismo, estos grupos contaron con una persona asignada a la coordinación de esta iniciativa. Como tal, la guía ofrecida por la coordinadora fue similar a la reportada por otros estudios (e.g., Allen, 2019; Lock et al., 2019; Smith et al., 2013) y permitió que se establecieran reglas generales de funcionamiento (e.g., lugar, horario y duración de las reuniones), se modelara la forma de dar y recibir retroalimentación, y se diera apoyo a la organización general de la agenda de cada grupo. Si bien no son generalizables por tratarse de un estudio de caso, nuestros resultados muestran que estos grupos presentan una opción para acompañar la escritura académico-científica de docentes experimentados y novatos que es fácil de implementar y sostenible a lo largo del tiempo (Aitchison \& Guerin, 2014). Dadas las condiciones actuales, resultado de la pandemia que estamos atravesando, futuros estudios podrían explorar si este tipo de dispositivo presenta similares ventajas en su formato virtual.

Asimismo, nuestros hallazgos también indicaron que los grupos de escritura facilitan un espacio donde sus 
participantes pueden aprender prácticas letradas dentro de un marco de compromiso mutuo a la vez que desarrollan su identidad como escritores académicos (e.g., Carr et al., 2020; Hicks et al., 2013). Sin embargo, esto solamente es posible si las instituciones universitarias están dispuestas a asignar los fondos necesarios de forma consistente para que se puedan mantener estos espacios orientados a incentivar y mejorar la actividad de escribir para publicar. Dado que muchos docentes universitarios enfrentan tensiones en cuanto a tener que cumplir con diferentes roles (e.g., docencia, gestión académica e investigación) (Sheridan et al., 2019), no es inusual que muchos de ellos produzcan sus publicaciones fuera del horario asignado a su trabajo. Como consecuencia, esta actividad termina siendo no remunerada (Aitchison \& Guerin, 2014). No obstante, como se mencionó en la introducción, las publicaciones en revistas de alto impacto favorecen no sólo a los docentes sino también a sus instituciones de pertenencia, en tanto constituyen un parámetro de evaluación de la calidad académica. Para superar estas limitaciones, creemos que es necesario que las universidades reconozcan tanto las actividades asociadas con la escritura, revisión y publicación de artículos dentro de los distributivos de los docentes investigadores, así como la necesidad de asignar horas de manera consistente para los encargados de implementar y coordinar iniciativas como la que aquí reportamos. Esperamos que los resultados de nuestra investigación informen no sólo acciones similares futuras en la Universidad de Cuenca y en otros ámbitos, sino que también contribuyan al fortalecimiento del campo de las pedagogías de la escritura en relación con el desarrollo profesional de los docentes e investigadores universitarios.

\section{AGRADECIMIENTO}

Los autores quisieran agradecer a los miembros de los grupos de escritura por haber contribuido con su perspectiva al igual que con su participación y entusiasmo para que este proyecto continúe.

\section{FINANCIAMIENTO}

Este trabajo ha sido financiado por la Dirección de Investigación de la Universidad de Cuenca (DIUC) como parte del proyecto de investigación "Análisis del funcionamiento de grupos de escritura de investigadores de la Universidad de Cuenca: interacción y percepciones".

\section{REFERENCIAS}

Aitchison, C., \& Guerin, C. (2014). Writing groups for doctoral education and beyond: Innovations in practice and theory. Abingdon: Routledge.

Allen, T. J. (2019). Facilitating graduate student and faculty member writing groups: experiences from a university in Japan. Higher Education Research and Development, 38(3), 435-449. https://doi.org/10.1080/07294360.2019.1574721.

Altbach, P. G. (2013). Advancing the national and global knowledge economy: the role of research universities in developing countries. Studies in Higher Education,
38(3), 316-330.

https://doi.org/10.1080/03075079.2013.773222

Antoniou, M., \& Moriarty, J. (2008). What can academic writers learn from creative writers? Developing guidance and support for lecturers in higher education. Teaching in Higher Education, 13(2), 157-167. https://doi.org/10.1080/13562510801923229

Bautista, A., Monereo, C., \& Scheuer, N. (2014). The peer review process as an opportunity for learning / $\mathrm{La}$ evaluación de pares como oportunidad para el aprendizaje. Infancia y aprendizaje / Journals for the Study of Education and Development, 37(4), 665-686. https://doi.org/10.1080/02103702.2014.977105

Bosanquet, A., McNeill, M., Huber, E., Cahir, J., \& Jacenyik-Trawoger, C. (2012). Reflection, speed dating, and word clouds: Evaluating a writing group for early-career researchers. Compendium, 2(5), 9-18. Recuperado de: https://ojs.library.dal.ca/C2/article/view/3841/3511

Carr, M., Clarkin-Phillips, J., Rinehart, K. E., Edwards, F., \& Ferrier-Kerr, J. (2020). Writing group commitment and caring: teacher educators talk about identities and agency in the Third Space of a writing group. Teacher Development, 24(5), 669-687. https://doi.org/10.1080/13664530.2020.1812708

Cassese, E. C., \& Holman, M. (2018). Writing groups as models for peer mentorship among female faculty in political science. American Political Science Association, 51(2), 401-405. https://doi.org/10.1017/S1049096517002049

Castelló, M. (Ed.). (2007). Escribir y comunicarse en contextos científicos y académicos. Conocimientos y estrategias. Barcelona: Graó.

Consejo de Educación Superior (CES). (2017). Reglamento de carrera y escalafón del profesor e investigador del Sistema de Educación Superior. Quito, Ecuador.

Chois-Lenis, P. M., Guerrero-Jiménez, H. I., \& Brambila-Limón, R. (2020). An analytical view of writing in graduate school: A review of documented practices in Latin America. Íkala, 25(2), 535-556. https://doi.org/10.17533/udea.ikala.v25n02a09

Colombo, L. (2013). Una experiencia pedagógica con grupos de escritura en el posgrado. Aula Universitaria, 15, 61-68. Recuperado de: http://bibliotecavirtual.unl.edu.ar/publicaciones/index.p hp/AulaUniversitaria/article/view/4368/6643

Colombo, L. (2017). Los grupos de escritura y el aprendizaje situado en el posgrado. Jornaleros, Revista Científica de Estudios Literarios y Lingüísticos, 3(3), 154-164. Recuperado de: https://ri.conicet.gov.ar/handle/11336/75731.

Colombo, L., \& Rodas, E. L. (2020). Interdisciplinarity as an opportunity in Argentinian and Ecuadorian writing groups. Higher Education Research and Development. 40(2), 207-219.

https://doi.org/10.1080/07294360.2020.1756750

Creswell, J. W. (2007). Qualitative inquiry \& research design: Choosing among five approaches ( $2 \mathrm{nd}$ ed.). Thousand Oaks, CA: Sage Publications.

De Feo, J. D., Kiliç, Z., \& Maseda, R. (2016). From productivity to process: Flipping the writing group. Academic Journal of Interdisciplinary Studies, 5(3), 
544-550. https://doi.org/10.5901/ajis.2016.v5n3s1p544

Denzin, N. K., \& Lincoln, Y. S. (Eds.). (2005). The Sage handbook of qualitative research ( $3 \mathrm{rd} \mathrm{ed}$.). Thousand Oaks, CA: Sage Publications.

Dwyer, A., Lewis, B., McDonald, F., \& Burns, M. (2012). It is always a pleasure: exploring productivity and pleasure in a writing group for early career academics. Studies in Continuing Education, 32(4), 129-44.

https://doi.org/10.1080/0158037X.2011.580734

Fajt, V., Gelvick, F. I., Loureiro-Rodríguez, V., Merton, P., Moore, G., Moyna, M. I., \& Zarestky, J. (2013). Feedback and fellowship. Stories from a successful writing group. En Geller, A. E. \& Eodice, M. (Eds.), Working with Faculty Writers (pp.163-173). Logan: Utah State UP.

Fallon, L. C., \& Whitney, A. E. (2016). "It's a two-way street": Giving feedback in a teacher writing group. Teaching/Writing: The Journal of Writing Teacher Education, 5(1), 1-16. Article 4. Recuperado de: https://scholarworks.wmich.edu/wte/vol5/iss1/4.

Feyen, J. (2020). Collaboration among disciplines and UEPs is essential to improve their teaching and research profile. Case Ecuador. Maskana, 11(2), 5-17. https://doi.org/10.18537/mskn.11.02.01

Feyen, J., Milia, M., Van Hoof, H. B., Quinde, K., Ochoa, V., Abril, V., \& Bojorque, J. (2016). Improving the publication visibility of Ecuador's higher education system. Maskana, 7(2), 113-125. https://doi.org/10.18537/mskn.007.002.08

Galligan, L., Cretchley, P., George, L., Martin, K., McDonald, J., \& Rankin, J. (2003). Evolution and emerging trends of university writing groups. Queensland Journal of Educational Research, 19(1), 28-41. Recuperado de: http://www.iier.org.au/qjer/qjer19/galligan.html

Gere, A. R. (1987). Writing groups: History, theory, and implications. Carbondale \& Edwardsville: Southern Illinois UP.

Gómez Nashiki, A., Jiménez-García, S. A., \& Moreles Vázquez, J. (2014). Publicar en revistas científicas, recomendaciones de investigadores de ciencias sociales y humanidades. Revista Mexicana de Investigación Educativa, XIX(60), 155-185. Recuperado de: https://www.redalyc.org/pdf/140/14029405008.pdf

Grant, B. (2006). Writing in the company of other women: Exceeding the boundaries. Studies in Higher Education, 31(4), 483-495. https://doi.org/10.1080/03075070600800624

Grant, B., \& Knowles, S. (2000). Flights of imagination: Academic women be(com)ing writers. International Journal for Academic Development, 5(1), 6-19. https://doi.org/10.1080/136014400410060

Guerin, C. (2014). The gift of writing groups: critique, community and confidence. En C. Aitchison \& C. Guerin (Eds.), Writing Groups for Doctoral Education and Beyond (pp. 138-141). Abingdon: Routledge.

Habibie, P., \& Hyland, K. (2019). Introduction: The risks and rewards of scholarly publishing. En P. Habibie \& K. Hyland (Eds.) Novice writers and scholarly publication: Authors, mentors, gatekeepers (pp.1-10). Switzerland: Palgrave Macmillan. doi:10.1007/978-3319-95333-5
Haas, S. (2014). Pick-n-Mix: a typology of writers' groups. En C. Aitchison \& C. Guerin (Eds.), Writing groups for doctoral education and beyond: Innovations in practice and theory (pp. 30-47). Abingdon: Routledge.

Hicks, T., Busch-Grabmeyer, E., Hyler, J., \& Smoker, A. (2013). Write, respond, repeat: A model for teachers' professional writing groups in a digital age. En K. E. Pytash, R. E. Ferdig \& T. V. Rasinski (Eds.), Preparing teachers to teach writing using technology (pp. 149-161). Pittsburg, PA: ETC Press.

Johnson, L., Roitman, S., Morgan, A., \& MacLeod, J. (2017). Challenging the productivity mantra: academic writing with spirit in place. Higher Education Research and Development, 36(6), 1181-1193. https://doi.org/10.1080/07294360.2017.1300140

Ley Organica de Educación Superior, LOES (2010). Asamblea Nacional del Ecuador.

Lee, I. (2013). Publish or perish: The myth and reality of academic publishing. Language Teaching, 47(2), 1-12. https://doi.org/10.1017/S0261444811000504

Lock, J., Kjorlien, Y., Tweedie, G., Dressler, R., Eaton, S.E., \& Spring, E. (2019). Advancing the writing of academics: Stories from the writing group. En N. Simmons \& A. Singh (Eds.), Critical Collaborative Communities (pp. 55-65). Koninklijke Brill NV. https://doi.org/10.1163/9789004410985_005

Marquez-Guzman, S. \& Gómez-Zermeño, M. G. (2018). Grupo virtual de escritura académica: Una einnovación para impulsar la publicación científica. Revista Mexicana de Investigación Educativa, 23(76), 203-227. Recuperado de:

http://www.scielo.org.mx/scielo.php?script=sci_abstra ct\&pid=S1405-66662018000100203\&lng= es\&nrm=iso

Maxwell, J. A. (2013). Qualitative research design: An interactive approach (3rd ed.). London: Sage.

Maxwell, J. A. \& Miller, B. (2008). Categorizing and connecting strategies in qualitative data analysis. En S. Hesse-Biber \& P. Leavy (Eds.), Handbook of Emergent Methods (pp. 461-477). New York: The Guilford Press

McGrail, M., Rickard, C., \& Jones, R. (2006). Publish or perish: A systematic review of interventions to increase academic publication rates. Higher Education Research \& Development, 25(1), 19-35. https://doi.org/10.1080/07294360500453053

Morales, J. (2013). La universidad investigadora. Revista de la Facultad de Ciencias Médicas de la Universidad de Cuenca, 3(11), 68-73.

Murray, R. (2013). 'It's not a hobby': Reconceptualizing the place of writing in academic work. Higher Education, 66, 79-91. https://doi.org/10.1007/s10734012-9591-7

Navarro, F. (2017). Estudios latinoamericanos de la escritura en educación superior y contextos profesionales: Hacia la configuración de un campo disciplinar propio. Lenguas Modernas, 50, 8-14. Recuperado de: https://lenguasmodernas.uchile.cl/index.php/LM/article /view/49247/51714.

Nygaard, L. (2015). Publishing and perishing: An academic literacies framework for investigating 
research productivity. Studies in Higher Education, 42(3), 519-532.

https://doi.org/10.1080/03075079.2015.1058351

Olszewska, K., \& Lock, J. (2016). Examining success and sustainability of academic writing: A case study of two writing-group models. Canadian Journal of Higher Education, 46(4), 132-145. https://doi.org/10.47678/cjhe.v46i4.186346

Plan Nacional de Desarrollo 2017-2021 - Toda una Vida. (2017). Ecuador.

Plummer, L., Pavalko, E., Alexander, J., \& McLeod, J. (2019) Faculty Writing Groups: A Tool for Providing Support, Community, and Accountability at MidCareer. En A. G. Welch, J. Bolin, \& D. Reardon (Eds) Mid-Career Faculty: Trends, Barries, and Possibilities (pp. 126-142). Boston: Brill.

Rijlaarsdam, G., \& van den Berg, H. (2006). Writing process theory: A functional dynamic approach. En C. A. MacArthur, S. Graham, \& J. Fitzgerald (Eds.), Handbook of writing research (pp. 41-53.). New York: The Guilford Press.

Rivera García, C. G., Espinosa Manfugás, J. M., \& Valdés Bencomo, Y. D. (2017). La investigación científica en las universidades ecuatorianas. Prioridad del sistema educativo vigente. Revista Cubana Educación Superior, 2, 113-125. Recuperado de: http://scielo.sld.cu/pdf/rces/v36n2/rces11217.pdf

Rodas Brosam, E., \& Colombo, L. (2018). Writing groups in Ecuador as support for academics on the road to publication. Revista Pucara 29, 147-167. https://doi.org/10.18537/puc.29.01.07

Seidman, I. E. (2006). Interviewing as qualitative research: A guide for researchers in education and the social sciences (3rd. ed.). New York: Teachers College Press.
Sheridan, A., O'Sullivan, J., Fisher, J., Dunne, K., \& Beck, W. (2019). Responding to institutional climate change in higher education: the evolution of a writing group to group peer mentoring. Gender and Education, 32(7), 891-907. https://doi.org/10.1080/09540253.2019.1594705

Smith, T., Molloy, J., Kassens-Noor, E., Li, W., \& Colunga, M. (2013). Developing a heuristic for multidisciplinary faculty writing groups. En A. E. Geller, \& M. Eodice (Eds.), Working with faculty writers (pp. 175-188). Salt Lake City: University of Utah Press.

Stensaker, B. (2017). Academic development as cultural work: responding to the organizational complexity of modern higher education institutions, International Journal for Academic Development, 23(4), 274-285. https://doi.org/10.1080/1360144X.2017.1366322

Sutherland, K. A. (2018) Holistic academic development: Is it time to think more broadly about the academic development project? International Journal for Academic Development, 23(4), 261-273. https://doi.org/10.1080/1360144X.2018.1524571

Sword, H., Trofimova, E., \& Ballard, M. (2018). Frustrated academic writers. Higher Education Research and Development, 37(4), 852-867. https://doi.org/10.1080/07294360.2018.1441811

Wilson, S., \& Cutri, J. (2019). Negating isolation and imposter syndrome through writing as product and as process: The impact of collegiate writing networks during a doctoral programme. En L. Pretorius, L. Macaulay, \& B. Cahusac de Caux (Eds.), Wellbeing in doctoral education: Insights and guidance from the student experience (pp. 59-76). Singapore: Springer. 